\title{
APORTE FISCAL DIRECTO PARA INSTITUCIONES DEL CONSEJO DE RECTORES DE LAS UNIVERSIDADES CHILENAS (1993-2008)
}

\section{Introducción}

Una de las maneras de clasificar las instituciones que componen el sistema universitario chileno es dividirlas en dos grandes grupos: universidades "tradicionales" y universidades "privadas". Las primeras son aquellas ocho que existían antes de la ley universitaria de 1980 más las que de ellas se derivaron. Las segundas nacen (o se reconocen) después de la dictación de dicha ley. De manera operacional, las universidades "tradicionales" pueden ser definidas como aquellas que forman parte del Consejo de Rectores de Universidades Chilenas $(\mathrm{CRUCH})$, organismo que se vincula con el Estado a través del Ministerio de Educación. En efecto, el ministro de Educación es el presidente del $\mathrm{CRUCH}$.

Las universidades pertenecientes al CRUCH reciben del Estado un subsidio establecido en la Ley de Presupuesto, denominado Aporte Fiscal Directo (AFD). Claramente, este es un aporte a la oferta en el sistema de educación superior y constituye un conjunto de recursos basales no sujetos al logro de alguna meta explícita. Sin embargo, la asignación de dicho aporte para cada una de las diversidades se rige por un algoritmo consistente en que el aporte del año presente (que podemos designar por $i+1$ ) se calcula manteniendo en un $95 \%$ la proporción recibida en años anteriores (i) y el 5\% se reasigna de acuerdo con un conjunto de indicadores preestablecidos. De esta manera, el AFD consta de una parte "histórica", podemos decir, y otra que podríamos llamar "por desempeño" o "variable". Este sistema de distribución y las características principales del sistema universitario chileno han sido ampliamente descritos en la literatura reciente (Bernasconi y Rojas, 2004; Brunner et al., 2005; Rappaport et al., 2004; Lavados, 2006). 
Los indicadores de desempeño considerados en la asignación del 5\% variable, se ponderan de acuerdo a los siguientes criterios:

i. Número de alumnos/número de carreras (pregrado): 1\%

ii. Número de alumnos/número de profesores (jornada completa equivalente, JCE): 15\%

iii. Número de profesores JCE con posgrado/número profesores JCE: $24 \%$

iv. Número de proyectos de investigación y desarrollo (concursos oficiales)/número de profesores JCE: 25\%

v. Número de publicaciones indexadas/número de profesores JCE: $35 \%$

Llamamos índice de desempeño, $\alpha_{i}^{j}$, a la fracción del 5\% (el porcentaje que corresponde a cada institución de ese 5\% para redistribuir) de la universidad $j$ el año $i$. Entonces la fracción del AFD total que corresponde a la misma institución al año siguiente, $X_{i+1}^{j}$, es la suma de la fracción recurrente del año anterior (ponderada por $p=0.95$ ) más lo logrado con índice de desempeño $\alpha_{i}^{j}$ (ponderado en $q=0.05)$. Es decir:

$$
X_{i+1}^{j}=p X_{i}^{j}+q \alpha_{i}^{j}
$$

La simple enumeración de los factores que inciden en la redistribución del AFD (todos en forma de ratio) permiten suponer que el Ministerio de Educación ha establecido una política en la cual es deseable un desempeño de las instituciones universitarias que privilegie la eficiencia docente (indicador ii), la calidad de los profesores (indicador iii) y la actividad de investigación (indicadores iv y v). La eficiencia en la formación de carreras pertinentes (indicador i) tiene una presencia porcentual marginal. Por otra parte, la asignación recurrente de los aportes indica una política de redistribución que debiera cambiar la asignación histórica (que es el punto de partida de la asignación del AFD). La combinación ponderada de los factores puede usarse para determinar un "índice de desempeño AFD" que varía cada año en cada universidad.

En el presente artículo se describirá el comportamiento temporal de estos índices de desempeño $\alpha_{i}^{j}$. En particular, trataremos 
de determinar si efectivamente la distribución ha cambiado con el tiempo. Usaremos los datos públicos de asignación de este aporte (Ministerio de Educación, 2008) entre 1993 y 2008 (1993 fue el año en que las universidades derivadas de la Pontificia Universidad Católica de Chile recibieron por primera vez AFD, completando así la recepción de esta asignación para la 25 universidades del $\mathrm{CRUCH}$ ). Usaremos los años de asignación aunque realmente representan el desempeño del año anterior. Desde 1993 hasta 2008 las 25 universidades tradicionales agrupadas en el $\mathrm{CRUCH}$ reciben este aporte; de este modo, la matriz de índices de desempeño tiene 25 columnas (instituciones) y 16 filas (años).

La consideración de fracciones (o porcentajes) nos permite hacer comparaciones y adelantar conclusiones sin considerar el gasto neto total.

\section{Análisis y discusión}

Los datos obtenidos para la distribución del AFD total entre 1993 y 2008 aparecen en el gráfico 1. Los índices de desempeño (los porcentajes del 5\% del AFD) se ilustran en una serie temporal en el gráfico 2, para lo mismos años. Se observa -a primera vistauna concentración en las universidades más antiguas del sistema universitario y lo mismo sucede para las fracciones AFD - 5\%. También, las distribuciones del ADF total y el AFD 5\% son muy similares entre los años considerados, con la excepción de variaciones significativas en dos casos: la disminución de la participación de la Universidad del Chile y el incremento de ésta por la Universidad de Talca. Estas instituciones son "anómalas" en el conjunto de datos considerados. 
Gráfico 1.

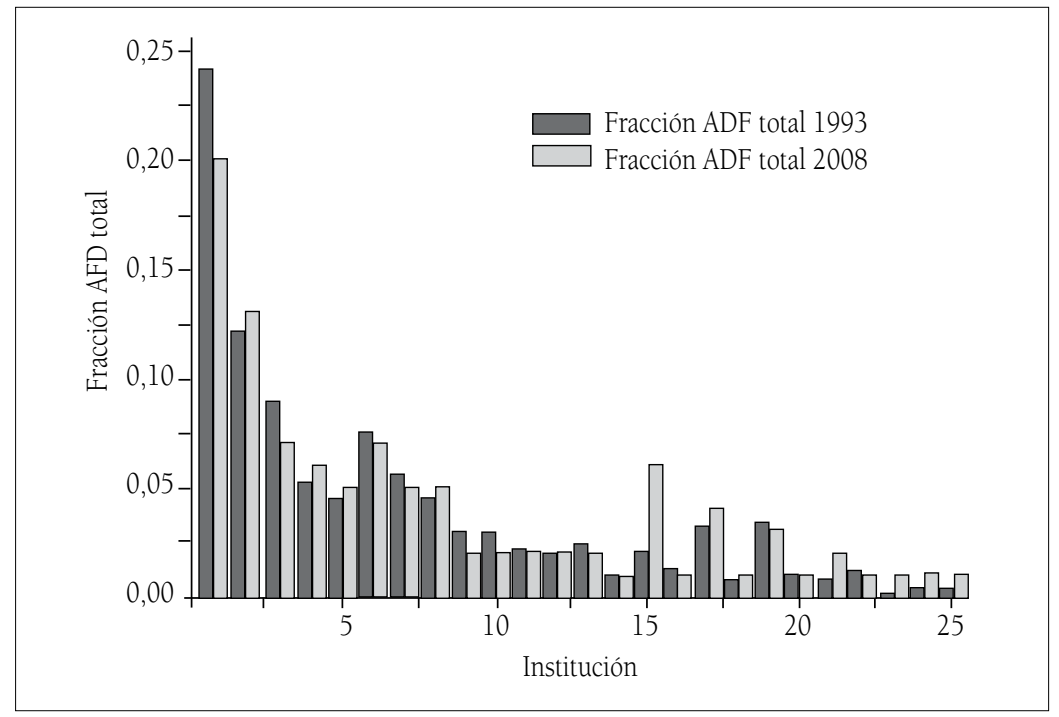

Gráfico 2.

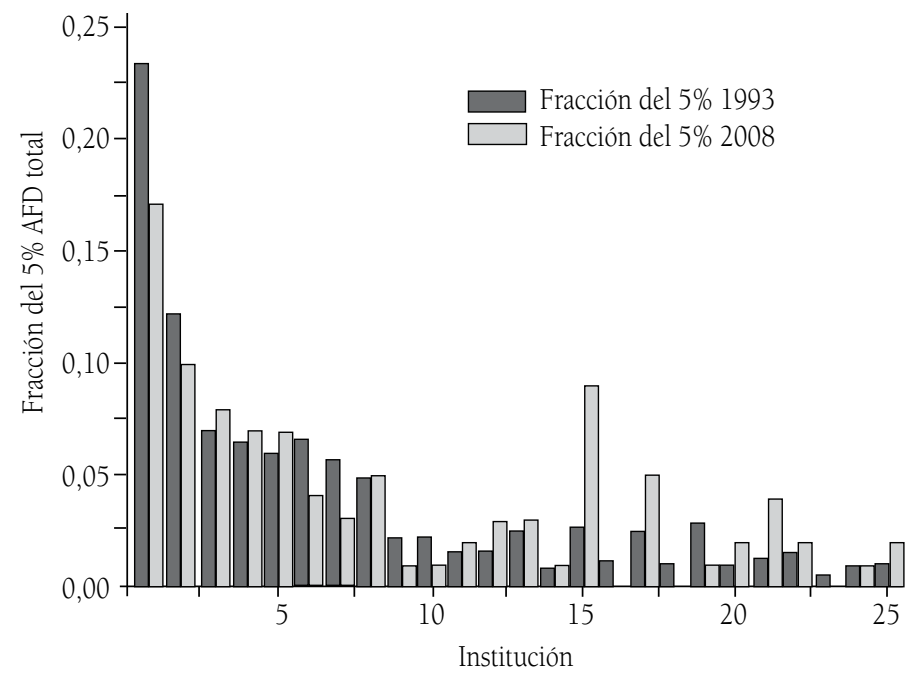

$\mathrm{Al}$ analizar las cifras en su conjunto, aparece claramente una asimetría en la distribución del AFD total y AFD - 5\%, situación que se mantiene en el tiempo. De este modo, la forma de redistribución del AFD no ha generado una repartición de los recursos que difiera significativamente en los años de aplicación de esta normativa. 
Un indicador usado con frecuencia para caracterizar la distribución del ingreso (entendido éste de manera genérica, aunque generalmente expresado como ingreso económico) es el índice de Gini (Gini, 1921; Xu, 2003). Si comparamos la distribución porcentual del 5\% del AFD en los años que analizamos encontramos un valor de 0,545 para 1999 y 0,486 para 2008. Las curvas de Lorenz (Lorenz, 1905) en que se basa este indicador se muestran en el gráfico 3. El índice de Gini tiene valores extremos de cero (perfecta equidad) y uno (inequidad total). Aunque la literatura econométrica relativa a la distribución comparada del ingreso en diversos países no entrega valores absolutos o limítrofes para una distribución equitativa o no, hay consenso en que un índice mayor que 0,5 demuestra desigualdad. En estos términos, puede concluirse que la disparidad de asignaciones del AFD entre las diversas universidades del CRUCH ha disminuido levemente en los años considerados.

Gráfico 3.

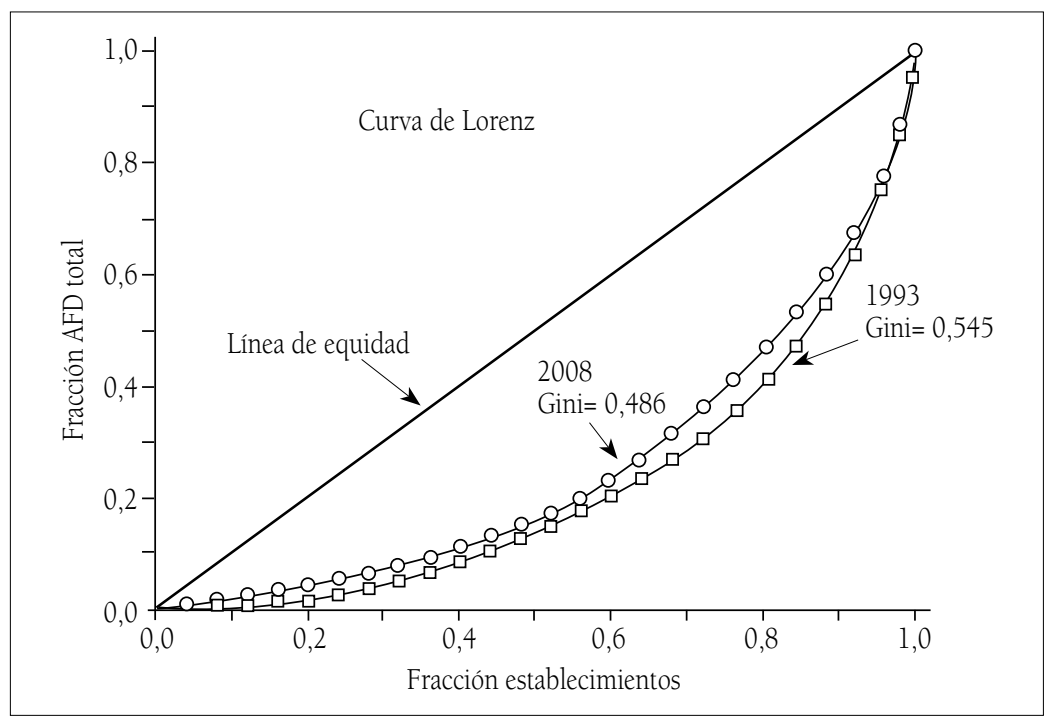

Para usar un ejemplo del ámbito económico, el índice Gini de distribución de los ingresos en Chile es 0,55 (año 2003) y el de Austria es 0,26 (año 2006). Determinar el valor del índice de Gini que separa la desigualdad de la igualdad es una materia en discusión, pero los índices citados son señales de una desigualdad en el ingreso para Chile y de una distribución más pareja para Austria. 
Otra cuestión, frecuentemente formulada y relacionada con el análisis anterior, es la permanencia en el tiempo de la asignación histórica inicial. Aunque claramente la distribución se ha modificado, el sistema en su conjunto no muestra una evolución apreciable. El diseño del sistema de asignación del 5\% de AFD ya incluye una dilución del estado final. Usando la Ec.1, es fácil ver que la parte histórica del AFD para cualquier institución, está dada por $p^{n} X_{0}$, donde $X_{0}$ es la fracción inicial y $n$ es el número de años transcurridos. Con $p=0,95$, esto significa que después de 15 años el efecto memoria llega al $46 \%$ y alcanza el 20\% luego de 30 años.

Hay que destacar que el AFD no es la única herramienta de política pública estatal. El Estado cuenta con otra serie de instrumentos para incentivar la mejoría del sistema universitario, disponibles no sólo para universidades pertenecientes al CRUCH. Entre ellos, el "Aporte Fiscal Indirecto" (AFI), los proyectos Mecesup, el sistema de becas y créditos, y variados proyectos de investigación, desarrollo e innovación administrados por la Comisión Nacional de Ciencia y Tecnología (CONICYT), los ministerios de Planificación, Agricultura, Educación, Salud, y Economía (proyectos Corfo, FIP), entre otros.

En resumen, se ha analizado la evidencia de los datos del índice de desempeño como fracción del 5\% del AFD para distribuir año a año. El índice de Gini, como indicador de equidad, muestra que el sistema evoluciona lentamente a una fórmula algo más igualitaria en el periodo de tiempo considerado. La evolución de este aporte ha cambiado sólo marginalmente las magnitudes asignadas a las universidades, con excepción de las dos universidades antes señaladas, y el sistema como conjunto resulta impermeable al efecto de las políticas que redistribuyen el AFD. Finalmente, la influencia de la distribución original de 1993 ha sido diluida y el efecto memoria ha disminuido sustancialmente.

El autor agradece la colaboración eficiente de L. Contreras en la obtención de los datos en que se basa este trabajo. 


\section{Referencias bibliográficas}

Bernasconi A. y Rojas F. (2004) Informe sobre la Educación Superior en Chile. Santiago de Chile: Editorial Universitaria.

Brunner J.J., Elacqua G., Tillet A., et al. (2005) Guiar el Mercado: Informe sobre la Educación en Chile. Sitio en Internet. Disponible en www.uai. cl (consultado agosto 2008).

Lavados J. (2006) Los negocios universitarios en el mercado del conocimiento. Santiago de Chile: LOM Editores.

Lorenz, M.O. (1905) Methods of measuring the concentration of wealth. Publications of the American Statistical Association, 9: pp. 209-219.

Ministerio de Educación. Chile. Sitio en Internet. Disponible en www.mineduc. cl/educacionsuperior (consultado agosto 2008).

Rappaport D., Benavente J.M. y Meller P. (2004) Rankings de universidades chilenas según los ingresos de sus titulados. Central Bank of Chile, Working Paper No 306. Sitio en Internet. Disponible en http://www.bcentral.cl (consultado agosto 2008).

$\mathrm{Xu}$, Kuan (2003) How has the literature on Gini's index evolved in the past 80 years? China Economic Quarterly, 2, pp. 757-778.

United Nations Development Program. Human Development Report 2007/2008. Sitio en Internet. Disponible en http://hdr.undp.org/en/ (consultado agosto 2008).

Recibido: 15 de octubre de 2008

Aceptado: 21 de enero de 2009 